\title{
A rare case of cardiac mass complicated with chronic thromboembolic pulmonary hypertension
}

\author{
xiaoli qin ${ }^{1}$, Weitao Liang ${ }^{1}$, and Zhong $\mathrm{Wu}^{1}$ \\ ${ }^{1}$ Sichuan University West China Hospital
}

August 23, 2020

\begin{abstract}
Cardiac mass is very rare. We reported a case of cardiac mass complicated with chronic thromboembolic pulmonary hypertension.
\end{abstract}

A rare case of cardiac mass complicated with chronic thromboembolic pulmonary hypertension

Running title: cardiac mass with pulmonary embolism

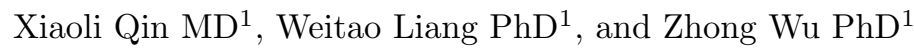

Co-first-author: Weitao Liang

${ }^{1}$ Department of Cardiovascular Surgery, West China Hospital, Sichuan University, Chengdu,

Sichuan, P.R.China.

Corresponding author: Zhong Wu

Postal Address: Department of Cardiovascular Surgery, West China Hospital, Sichuan

University; Chengdu, Sichuan, P.R. China, 610000;

Tel.: +86-028-85422897; Fax: +86-028-85422897; E-mail: wuzhong_71@163.com

Abstract

Cardiac mass is very rare. We reported a case of cardiac mass complicated with chronic thromboembolic pulmonary hypertension.

Keywords: cardiac mass, intracardiac thrombosis, pulmonary embolism

A 41-year-old man presented with an eight-month history of chest tightness and dyspnea. Transthoracic echocardiography (TTE) revealed a $4 * 2 \mathrm{~cm}$ mass attached to the tricuspid valve chordae and massive tricuspid regurgitation (Figures 1A-1C). The systolic tricuspid pressure gradient was $111 \mathrm{mmHg}$. Pulmonary computed tomography angiography demonstrated a filling defect located in the right ventricle, left and right pulmonary artery, and their distal parts (Figures $1 \mathrm{D}$ and $1 \mathrm{E}$ ). The laboratory test and lower extremity venous ultrasound revealed no abnormalities. The patient received the surgery and the intraoperative transesophageal echocardiography (TEE) showed the mass was attached to the tricuspid chordae tendineae (Figure $1 \mathrm{~F}$ ). After median sternotomy, cardiopulmonary bypass, and right atriotomy, an irregular-shaped mass was discovered and its pedicle was attached to the chordae tendineae (Figure 1G). We excised the mass and pulmonary embolism completely and performed pulmonary endarterectomy (Figures 1H and 1I). The postoperative period was uneventful. The histopathological examination of the mass revealed fibrous 
exudates and necrosis with focal calcification. The study was approved by our Institutional Review Board and oral informed consent was obtained from patient. There was no tumorigenesis and it was more like an organized thrombus. However, in our case, there was a pedicle of the mass attached to the chordae tendineae. Cardiac mass in Behçet's disease was reported and pathological examination was similar to our case. But there were no other positive symptoms of Behçet's disease in our case. References

1. Yue C, Li J, Li M, Zhang F, Zhao D, Cui Q. Cardiac mass in Behçet's disease. Clin Exp Rheumatol. 2012;30(3 Suppl 72):S27-S31.

2. Mogulkoc N, Burgess MI, Bishop PW. Intracardiac thrombus in Behçet's disease: a systematic review. Chest. 2000;118(2):479-487. doi:10.1378/chest.118.2.479.

Figure 1 A-C, Transthoracic echocardiography shows a $4 * 2 \mathrm{~cm}$ mass attached to the tricuspid valve chordae and massive tricuspid regurgitation; D and E, Pulmonary computed tomography angiography demonstrates a filling defect located in the right ventricle, left and right pulmonary artery, and their distal parts; F, Transesophageal echocardiography shows the mass attached to the tricuspid chordae tendineae; G-I, Removal of the mass and pulmonary embolism. LA, left atrium; LV, left ventricle; RA, right atrium; RV, right ventricle
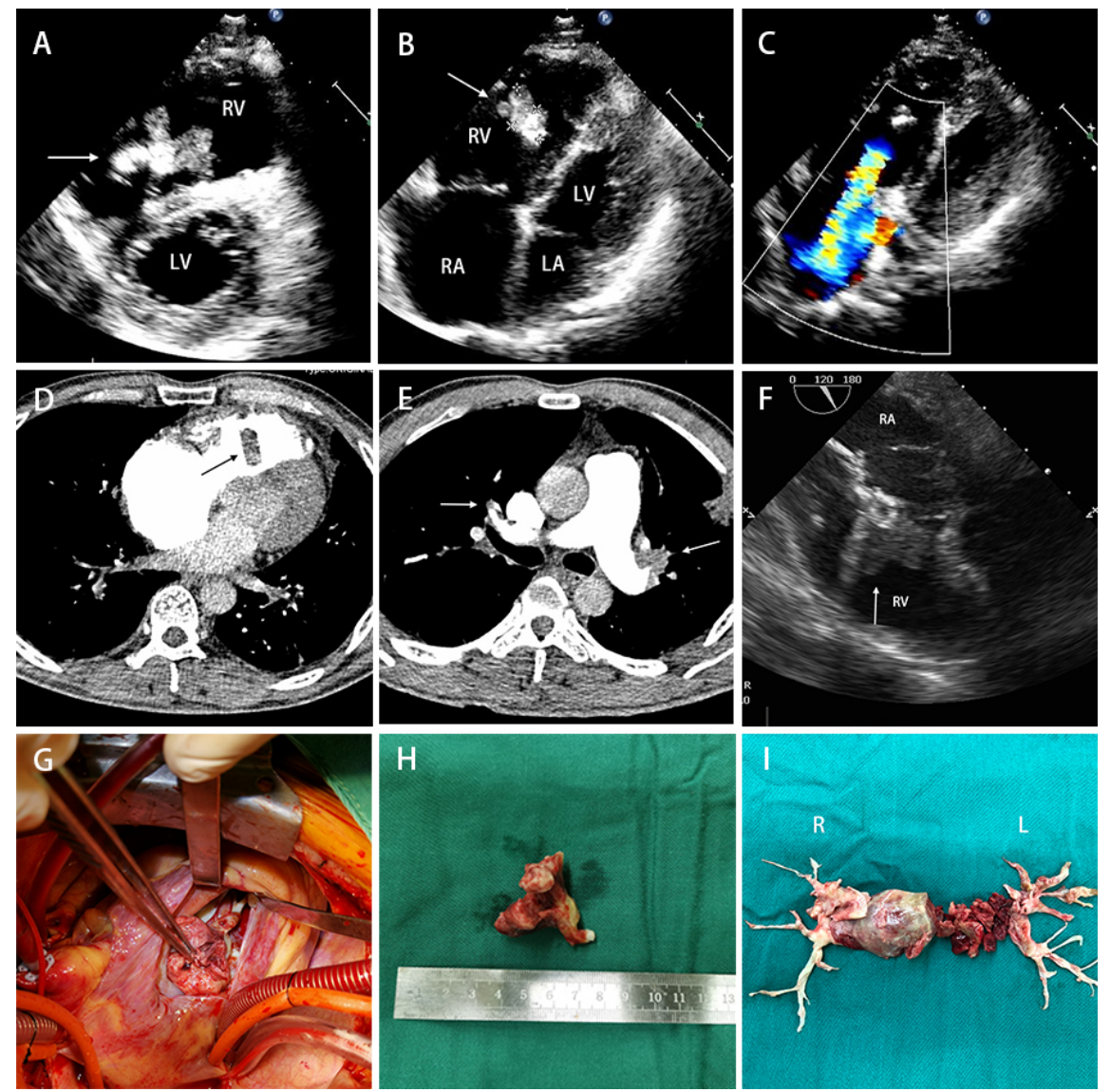